\title{
Socioeconomic Factors Associated with Poor Prognosis in Patients with Alcoholic Liver Cirrhosis
}

\section{Mio Kushibuchi ( $\square$ mio.kushibuchi@marianna-u.ac.jp )}

Kawasaki Municipal Tama Hospital

\section{Chiaki Okuse}

Kawasaki Municipal Tama Hospital

\section{Kenya le}

Kawasaki Municipal Tama Hospital

Kotaro Matsunaga

Kawasaki Municipal Tama Hospital

\section{Tomoya Tsuchida}

St. Marianna University School of Medicine

Iori Motohashi

Kawasaki Municipal Tama Hospital

\section{Masanori Hirose}

St. Marianna University School of Medicine

\section{Takuya Otsuki}

Kawasaki Municipal Tama Hospital

\section{Mari Aihara}

Kawasaki Municipal Tama Hospital

\section{Takahide Matsuda}

St. Marianna University School of Medicine

\section{Research Article}

Keywords: socioeconomic status, alcohol, liver cirrhosis, mortality

Posted Date: January 11th, 2022

DOI: https://doi.org/10.21203/rs.3.rs-1235046/v1

License: (9) This work is licensed under a Creative Commons Attribution 4.0 International License. Read Full License 


\section{Abstract}

Background: Alcohol liver cirrhosis is a life-threatening condition, especially if alcohol cessation is not accomplished. Past studies have shown that alcohol abuse is closely linked to low socioeconomic status and social marginalization. Public assistance (PA), or Seikatsu-hogo, a Japanese public assistance ensuring medical care to low-income population, was employed as a proxy for social marginalization. This study aims to investigate the prognostic effect of being a PA recipient on overall mortality in patients with alcoholic cirrhosis.

Methods: Patients diagnosed as alcoholic liver cirrhosis in a community hospital between 2006 to 2017 were included in this retrospective cohort study. Baseline demographics and mortality data were extracted from electronic health records. Cirrhosis severity at baseline was measured by mean model for end-stage liver disease (MELD) score and Albumin-Bilirubin (ALBI) score. Primary outcome was survival probability obtained by the Kapan Meier method and Cox proportional hazards regression.

Results: 244 participants were included, among which 62 were PA recipients. Baseline cirrhosis severity score was not different between the two groups. Incidence proportion for overall mortality was $48.4 \%$ and $31.9 \%$ for PA recipients and non-PA recipients, respectively $(p=0.002)$. In cox regression model, adjusted for age, ALBI score and HCV infection, hazard ratio for PA reception was1.57 (95\% Cl: 0.97-2.5, p=0.06).

Conclusions: Being a PA recipient may be a poor prognostic factor of mortality in patients with alcoholic liver cirrhosis.

\section{Introduction}

Cirrhosis contributes to $85 \%$ of alcohol-related deaths worldwide [1]-3 million deaths annually according to World Health Organization[2]. A previous study has revealed that five-year mortality of alcoholic cirrhosis was $58 \%$, with complications such as variceal bleeding and encephalopathy making the risk double-fold [3]. In Japan, annual age-standardized deaths from cirrhosis for men is 10.9 per 100 thousand individuals, and $67.8 \%$ is attributed to alcohol consumption [4].

Socioeconomic status (SES) refers to an individual's position in society; SES is established by a combination of educational, occupational, and economic criteria. SES, together with marital status, largely influences cirrhosis mortality. Regarding liver disease focusing on alcoholic etiology, a study from US have shown that patients with lower education, lower income, and unemployed had higher mortality than those better-off or employed[5]. In a study of Danish cirrhosis patients in which $85 \%$ of patients reported alcohol abuse, disabled pensioners, compared to employed, had 1.35 times higher mortality among cirrhosis patients in Denmark. Divorced had 1.22 times higher mortality than married [6]. In a study of cirrhosis from Sweden, in which $51 \%$ of cirrhosis was alcoholic, low- and middle-skilled workers, compared to professionals, had 1.85 times higher mortality [7]. 
Low SES individuals in Japan are warranted minimum living costs through a national Public Assistance (PA) system called Seikatsu- Hogo. PA system is the safety net of Japanese welfare system, thus those injured or sick without premium payment, or older population without sufficient pension benefits relies on PA programs [8]. PA consists of income security, employment support, and in-kind supply of medical and long-term care, financed by taxation. Therefore, Public Assistance beneficiaries can receive the same medical service as any other Japanese citizen, without any out-of-pocket payment. Previous studies in the US have shown that Medicaid and other health insurance schemes have contributed to improvements in access to health services, health status and quality of life in low-income populations[9,10]. However, no previous study up to our knowledge has investigated the prognostic effect of being a PA recipient in Japan.

Association between cirrhosis mortality and SES, especially employment and education, have been discussed. However, few have focused on alcoholic cirrhosis. Furthermore, up to our knowledge, previous studies have not taken clinical features such as cirrhosis severity and viral coinfection into account. Evidence regarding mortality of PA recipients in Japan is rare, and no previous study have revealed mortality hazard ratio of PA recipients in Japan. The aim of this study was therefore to investigate whether being a PA beneficiary was a poor prognostic factor for mortality in Japanese patients with alcoholic liver cirrhosis.

\section{Materials And Methods}

\section{Study population and inclusion criteria}

727 patients were diagnosed as liver cirrhosis (LC) in our institution during years 2006 to 2017. Our institution is a public hospital located in suburban area of Tokyo metropolitan area, with 376 beds and an outpatient ward. Etiology of LC was investigated by trained hepatologists by laboratory findings such as low Platelet and Albumin, and imaging test including ultrasound, computed tomography, or magnetic resonance image. 258 participants that had had history of excessive alcohol consumption and were diagnosed as alcoholic LC by the hepatologists were left. 12 patients that had only visited our outpatient ward once, and 2 that further medical record review revealed no presence of alcoholic LC were excluded from the analysis. Finally, a total of 244 patients were left and included for the present study. Inclusion and exclusion flowchart of participants is described in figure 1.

The research protocol was approved by Institutional Review Board of St Marianna University Hospital (approval number 4995).

\section{Basic demographics}

Basic demographics at point of diagnosis were obtained through medical record review. Age, sex, body mass index (BMI) calculated by height and weight, chief complaint at first hospital visit, and emergency hospitalization were recorded. Coexistence of viral hepatitis (HBV and HCV), and laboratory findings 
including Asparate aminotransferase (AST), Alanine aminotransferase (ALT), Creatinine, total Bilirubin, Albumin, Prothrombin time- international normalized ratio (PT-INR), and Platelet count, were obtained from medical record review.

Liver cirrhosis severity was measured by MELD score [11] and albumin-bilirubin (ALBI) score [12]. MELD score is an objective scoring calculated by creatinine, total Bilirubin, Albumin and PT-INR [11]. Whether the discrimination power of MELD score in predicting mortality is superior to that of Child Pugh Score, a classical scoring for liver cirrhosis, is controversial; however, a systematic review in 2016 reports that MELD score is as reliable a prognostic score as the Child Pugh score [13]. A study conducted in 2019 reported ALBI to be the optimal predictor of mortality compared to MELD, MELD-Na and Child Pugh [14]. Furthermore, a study among Asians have reported that ALBI was provided particularly reliable prediction for short-term outcomes, whereas the MELD score was better in terms of assessing long-term outcomes [15]. Considering that Child Pugh scoring is rather subjective in terms of ascites and encephalopathy scoring, we employed the MELD and ALBI scores in the current study.

Regarding socioeconomic status, whether the participant was a PA beneficiary was obtained from insurance information in medical record. Residing alone and having no key person (a family member or someone in close relationship to contact in case of emergency) were other SES measurements obtained. Information on SES variables mentioned above were obtained from medical record of the participants.

\section{Outcome measurements}

The primary outcome for our study was survival time after diagnosis of alcoholic liver cirrhosis. The patients were followed until death, dropout or continuous outpatient visit at March 2020 or later, whichever came first. Data for those that death was not recorded (either dropped out or were continuing hospital visit) was handled as censored data.

As secondary outcome for our study, the incidence of ascites, esophageal varices, hepatocellular carcinoma (HCC), encephalopathy and spontaneous bacterial peritonitis (SBP) were obtained. Presence of abovementioned complications observed at our hospital at any point during follow up period was counted as positive.

\section{Statistical analysis}

Basic demographics were depicted using median and interquartile range, or by percentage, depending on whether the variable was linear or bivariate. In order to test differences between the two subgroups, Chi squared analyses were applied for binary variables. Mann Whitney $\mathrm{U}$ tests were applied for continuous variables since all continuous variables had non-normal distribution. We computed survival probabilities using the Kapan Meier method and used Cox proportional hazards regression to estimate hazard ratios. Using the Schoenfeld residuals, we determined that hazard ratios were constant over the follow-up time. 
Mean survival time, survival rate at median follow-up, and five-year survival rate was obtained from survival curve for the two subgroups. Statistical difference of crude hazard ratio for being a PA recipient was obtained by Log rank test. Then, we included receiving PA, together with age, HCV infection, and ALBI score in one cox hazard model to obtain the adjusted hazard ratio. Significance level was set at the alpha value of 0.05 . All statistical analysis was computed using STATA version 16 software (Stata Corporation, College Station, TX, USA).

\section{Results}

244 patients met our inclusion criteria, of whom 62 (26.1\%) were PA recipients. 209 (80\%) were men, and median age was 61.8 [60.3-63.3]. Median follow-up time was 819 days. During a total follow-up time of 9100 months of time at risk, 88 patients (33.6\%) deceased. Basic demographics are shown in Table 1. Percentage of those living alone and those without a key person greatly differed between PA recipients and non-PA recipients $-74.2 \%$ versus $28.6 \%$ ( $p<0.001)$, and $29.1 \%$ versus $7.1 \%(p<0.001)$, respectively. As for clinical parameters at time of diagnosis, mean MELD score and ALBI score was 8.19 [7.40-8.98] and -1.92 [-2.04- -1.83], among which neither was significantly different between the two subgroups. No participant had HBV infection, thus only coexistence of HCV infection is reported in our results table. $24.4 \%$ of overall patients required emergent admission during the study period. The most common chief complaint was asymptomatic liver dysfunction (34.0\%). Other common symptoms were abdominal distension and edema (19.5\%), fatigue and immobility (7.6\%), gastrointestinal gastric and variceal bleeding (9.2\%), among others (Supplementary table 1 ).

\section{Table 1}

basic demographics of the participants, described by median ( $25 \%$ to $75 \%$ interquartile range) or number (percentage). P value was obtained by Mann Whitney U-test for medians, and chi-squared analysis for percentages. Abbreviations: KP, key person; BMI, body mass index; DM, diabetes mellitus; HCV, hepatitis C virus; AST, asparate aminotransferase; ALT, alanine transaminase; MELD, model for end-stage liver disease; $\mathrm{ALBI}$, albumin-bilirubin; PA, public assistance; $n$, number 
subgroup by public assistance

$\begin{array}{llll}\begin{array}{l}\text { Overall } \\ (n=244)\end{array} & \text { Non-PA } & \text { PA } & \text { p value }\end{array}$

\section{Demographics}

\begin{tabular}{|c|c|c|c|c|}
\hline Age & $\begin{array}{l}62 \\
{[54-70]}\end{array}$ & $\begin{array}{l}63 \\
{[54-72]}\end{array}$ & $\begin{array}{l}59 \\
{[53-67]}\end{array}$ & 0.05 \\
\hline Male & $209(79.8 \%)$ & $156(85.7 \%)$ & $53(85.5 \%)$ & 0.96 \\
\hline Living alone & $98(37.4 \%)$ & $52(28.6 \%)$ & $46(74.2 \%)$ & $<0.001$ ** \\
\hline No KP & $31(11.8 \%)$ & $13(7.14 \%)$ & $18(29.1 \%)$ & $<0.001$ ** \\
\hline Nursing care & $63(42.1 \%)$ & $43(23.6 \%)$ & $20(32.3 \%)$ & 0.31 \\
\hline BMI & $\begin{array}{l}22.0 \\
{[19.7-24.5]}\end{array}$ & $\begin{array}{l}21.8 \\
{[19.7-24.6]}\end{array}$ & $\begin{array}{l}22.2 \\
{[20.3-24.4]}\end{array}$ & 0.86 \\
\hline \multicolumn{5}{|c|}{ Presentation at first visit } \\
\hline Diabetes Mellitus & $70(26.7 \%)$ & $49(26.9 \%)$ & $21(33.9 \%)$ & 0.54 \\
\hline HCV infection & $52(19.8 \%)$ & $31(17.0 \%)$ & $21(33.9 \%)$ & $0.005 * \star$ \\
\hline AST & $\begin{array}{l}64 \\
\text { [38-105] }\end{array}$ & $\begin{array}{l}69 \\
{[40-107]}\end{array}$ & $\begin{array}{l}54 \\
{[34-97]}\end{array}$ & 0.06 \\
\hline ALT & $\begin{array}{l}37 \\
{[24-63]}\end{array}$ & $\begin{array}{l}39 \\
{[26-66]}\end{array}$ & $\begin{array}{l}29 \\
{[21-52]}\end{array}$ & $0.03 *$ \\
\hline Total Bilirubin & $\begin{array}{l}1.55 \\
{[0.9-3.00]}\end{array}$ & $\begin{array}{l}1.55 \\
{[0.9-3.0]}\end{array}$ & $\begin{array}{l}1.55 \\
{[0.9-2.8]}\end{array}$ & 0.69 \\
\hline Albumin & $\begin{array}{l}3.5 \\
{[2.9-3.9]}\end{array}$ & $\begin{array}{l}3.5 \\
{[3.0-3.9]}\end{array}$ & $\begin{array}{l}3.5 \\
{[2.8-3.8]}\end{array}$ & 0.53 \\
\hline PT-INR & $\begin{array}{l}1.09 \\
{[1.00-1.23]}\end{array}$ & $\begin{array}{l}1.08 \\
{[1.00-1.24]}\end{array}$ & $\begin{array}{l}1.11 \\
{[1.00-1.22]}\end{array}$ & 0.92 \\
\hline Platelet & $\begin{array}{l}11.3 \\
{[8.0-16.1]}\end{array}$ & $\begin{array}{l}11.0 \\
{[7.9-16.6]}\end{array}$ & $\begin{array}{l}13.1 \\
{[9.2-16.0]}\end{array}$ & 0.34 \\
\hline
\end{tabular}

\section{Cirrhosis severity at first visit}

$\begin{array}{lllll}\text { MELD score } & 7.0 & 7.0 & 8.0 & 0.44 \\ & {[3.0-12.0]} & {[3.0-12.0]} & {[4.0-13.0]} & \end{array}$

\begin{tabular}{lllll} 
ALBI score & -2.03 & -2.06 & -1.97 & 0.65 \\
\hline & {$[-2.48--1.42]$} & {$[-2.48--1.42]$} & {$[-2.50--1.43]$} &
\end{tabular}

ALBI Grade

\begin{tabular}{lllll} 
Grade冈 & $45(17.2 \%)$ & $32(17.6 \%)$ & $12(21.0 \%)$ & \multirow{2}{*}{0.78} \\
\cline { 1 - 3 } Grade2 & $142(54.2 \%)$ & $108(59.3 \%)$ & $34(54.8 \%)$ &
\end{tabular}


Grade3

$19(7.25 \%)$

$1(0.55 \%)$

$0(0 \%)$

Hospitalization

Emergent

$64(24.4 \%)$

$46(25.3 \%)$

$18(29.0 \%)$

0.577

Occurrence of primary and secondary outcomes are shown in Table 2. Mortality incidence proportion during the observed period was $33.6 \%$ overall, and $48.4 \%$ versus $31.9 \%(p=0.02)$ for PA recipients and non-PA recipients. The overall rate of treatment dropout was $18 \%$; it did not differ between the subgroups. Death causes included liver-related causes such as HCC, acute on chronic liver failure and varices rupture. Occurrence of complications-ascites, varices, HCC, SBP and encephalopathy-were not different among the two groups.

\section{Table 2}

Primary and secondary outcomes for PA recipients and non-PA recipients. Abbreviations: CPA, cardiopulmonary arrest; $\mathrm{HCC}$, hepatocellular carcinoma; SBP, spontaneous bacterial peritonitis; PA, public assistance; $n$, number. 
subgroup by public assistance

$\begin{array}{llll}\begin{array}{l}\text { Overall } \\ (n=234)\end{array} & \begin{array}{l}\text { Non-PA } \\ (n=173)\end{array} & \text { PA } & \text { p value } \\ & (n=61) & \end{array}$

\section{Mortality}

\begin{tabular}{|c|c|c|c|c|}
\hline Incidence proportion (\%) & $88(33.6 \%)$ & $58(31.9 \%)$ & $30(48.4 \%)$ & $0.02 *$ \\
\hline median survival time & $\begin{array}{l}2104 \\
{[1589-3563]}\end{array}$ & $\begin{array}{l}2889 \\
{[1692-N / A]}\end{array}$ & $\begin{array}{l}1559 \\
{[1589-3563]}\end{array}$ & N/A \\
\hline survival rate at median follow up (\%) & $\begin{array}{l}75.3 \% \\
{[68.7-80.7]}\end{array}$ & $\begin{array}{l}76.6 \% \\
{[68.8-82.7]}\end{array}$ & $\begin{array}{l}69.9 \% \\
{[56.0-80.1]}\end{array}$ & 0.28 \\
\hline five year survival rate & $\begin{array}{l}54.2 \rrbracket \\
{[45.6-62.0]}\end{array}$ & $\begin{array}{l}57.8 \% \\
{[47.7-66.6]}\end{array}$ & $\begin{array}{l}45.2 \% \\
{[29.2-60.6]}\end{array}$ & 0.07 \\
\hline \multicolumn{5}{|l|}{ treatment adherence } \\
\hline dropout rate & $48(18.3 \%)$ & $38(20.9 \%)$ & $10(16.3 \%)$ & 0.08 \\
\hline \multicolumn{5}{|l|}{ death cause } \\
\hline encephalopathy & $11(4.2 \%)$ & $5(2.7 \%)$ & $6(9.7 \%)$ & \\
\hline $\mathrm{HCC}$ & $14(5.3 \%)$ & $12(6.6 \%)$ & $2(3.2 \%)$ & \\
\hline HCC rupture & $11(4.2 \%)$ & $11(6.0 \%)$ & 0 & \\
\hline liver failure & $15(5.3 \%)$ & $10(5.5 \%)$ & $4(6.5 \%)$ & \\
\hline Other carci & $7(2.7 \%)$ & $5(2.8 \%)$ & $2(3.2 \%)$ & \\
\hline SBP & $2(0.8 \%)$ & 0 & $2(3.2 \%)$ & \\
\hline Sepsis/infection & $4(1.5 \%)$ & $2(1.1 \%)$ & $2(3.2 \%)$ & \\
\hline Varix rupture & $11(4.2 \%)$ & $6(3.3 \%)$ & $5(8.1 \%)$ & \\
\hline Others & $12(4.6 \%)$ & $7(3.9 \%)$ & $5(8.1 \%)$ & \\
\hline
\end{tabular}

Complications

\begin{tabular}{lllll} 
ascites & $203(83.2 \%)$ & $152(83.5 \%)$ & $51(82.3 \%)$ & 0.81 \\
\hline varix & $147(60.3 \%)$ & $112(61.5 \%)$ & $35(56.5 \%)$ & 0.55 \\
HCC & $97(39.8 \%)$ & $73(40.1 \%)$ & $24(38.7 \%)$ & 0.87 \\
SBP & $17(6.97 \%)$ & $11(6.04 \%)$ & $6(9.68 \%)$ & 0.32 \\
encephalopathy & $66(27.1 \%)$ & $48(26.4 \%)$ & $18(29.0 \%)$ & 0.65
\end{tabular}

Figure 2 shows the Kaplan Meier survival estimates for the PA recipients and non-PA recipients. Log Rank test showed no significant difference in survival rate between the two groups $(p=0.127)$. Median survival time were 51.9 months and 96.3 months for PA recipients and non-PA recipients, respectively. 
Survival rate at median follow up (819 days) were $66.9 \%$ and $76.6 \%$, respectively. Five-year survival rate was $45.2 \%$ and $57.8 \%$.

Cox proportional hazard model was applied to calculate hazard ratio of PA recipients, adjusting for age, HCV infection and ALBI score. Results are shown in Table 3. PA recipients had higher risk compared to non-recipients with hazard ratio of 1.57 [Cl: $0.97-2.53, p=0.06]$. In the multivariable analysis, age and ALBI scores were independent predictors of mortality, with hazard ratio 1.04 [Cl: $1.02-1.06, p<0.001]$ and 1.64 [Cl: 1.28-2.10, $p=0.001]$, respectively.

Table 3

Cox proportional hazard model of with age, HCV infection, ALBI and receiving PA included in the multivariate model. Abbreviations: HR, hazard ratio; $95 \mathrm{Cl}, 95$ percent confidence intervals; HCV, hepatitis $C$ virus; ALBI, Albumin-bilirubin; PA, public assistance.

\begin{tabular}{llll} 
& Cox HR & $95 \mathrm{Cl}$ & $\mathrm{p}$ value \\
\hline age & 1.04 & $1.02-1.06$ & $<0.001$ ** \\
\hline HCV infection & 1.3 & $0.80-2.12$ & 0.29 \\
\hline ALBI score & 1.64 & $1.28-2.10$ & 0.001 ** \\
\hline receiving PA & 1.57 & $0.97-2.53$ & 0.06
\end{tabular}

\section{Discussion}

In this retrospective cohort study of 244 patients with alcoholic liver cirrhosis, we found that PA recipients tended to have higher hazard ratio for overall death than non- PA recipients after adjusting for age, HCV infection and ALBI score. Basic demographics such as BMI and concurrence of Diabetes Mellitus did not differ between the two groups; however, social isolation as measured by residing alone and having no key person were remarkably higher among the PA recipients. Baseline severity of liver dysfunction measured by MELD score and ALBI score, as well as proportion of patients requiring emergent hospitalization at first presentation were not higher among PA recipients.

Owing to the public assistance system, low-income individuals in Japan have no financial barriers to healthcare services. However, non-financial barriers such as upstream determinants of health that is closely related to low socioeconomic status, may lead to higher mortality in the poor despite the financial and medical assistance provided [16]. We hypothesized that if receiving PA and overall survival is not associated, low SES may not be homogenously associated with lower health status as in most countries, thanks to the assistance system in Japan. On the other hand, if PA beneficiaries' survival is relatively shorter, effect of low SES on mortality cannot be subsided by financial warrant. 
Our study revealed a tendency of higher mortality among those receiving PA (adjusted $\mathrm{HR} 1.57(95 \% \mathrm{Cl}$ 0.97-2.53, $p=0.06)$ ). Previous study by Jepsen et al has shown that poorer cirrhosis prognosis among divorced and unmarried, compared to married participants [6]. Our study concords with this result since PA recipients were more likely to be living alone or have no key person, and PA recipients tended to have poorer prognosis. This may be due to their lack of social support such as family members or workplace communities [17].

Furthermore, MELD score and need for emergent hospitalization did not differ among PA recipients and non-PA recipients at the point of diagnosis. Treatment dropout rate was also indistinguishable among the two groups. A literature review shows that socioeconomically deprived older population has worse access to care [18]. In our study, neither cirrhosis severity at the point of diagnosis nor dropout rate were different between PA recipients and non-PA recipients-in other words, healthcare access barrier was not observed at both point of diagnosis and at treatment continuum among cirrhosis patients that visited our hospital.

Our study has several clinical implications. Abovementioned results demonstrate that although neither cirrhosis severity at baseline nor dropout rate were different among the two groups, PA recipients had poorer prognosis. Several reasons for this health gap can be speculated, but one possible explanation may be that PA recipients experienced more hardship quitting alcohol. There is explicit evidence that shows strong relationship between alcohol-related death and low SES [1,19]. Although we couldn't measure severity of alcohol addiction in our study, it may be possible that PA recipients had lower alcohol cessation rate after diagnosis, probably due to family or social support. If so, clinicians should provide appropriate support for patients with alcoholic cirrhosis to improve their health-related behaviors. The results also imply that providing alcohol cessation information, and connecting low SES patients to social support such as mutual self-help groups may improve PA recipients' prognosis.

In our cox hazard model, hazard ratio for PA recipient was not statistically significant, although difference in mortality was conspicuous in the survival curve. We assume that this was due to lack of statistical power. As an attempt to decrease the number of censored data, we conducted a follow-up written survey to the 129 participants that were censored. We did not have the post address for 5 of the participants. Among 124 surveys sent, 65 of the address were invalid, thus we could not contact the patient. Only 20 had replied, and among them, 9 had agreed to participate in the survey. We assumed that there would be a selection bias to include the nine valid answers in our study, and that the population would not be homogenous with the medical record-based dataset. Therefore, we decided to exclude the follow-up survey results. The high percentage of uncontactable participants may be reflecting the social isolation of these population.

Other limitations to our study were that it was single-centered, and we did not have as much participants that would have enabled us to include more confounders in our multivariable analyses. We could not take into account comorbidities other than HCV infection; deaths may have occurred due to other critical illnesses. Also, we could only measure limited factors of SES, and more in-depth investigation on discrete 
SES components is needed to unveil which upstream drivers of health is strongly affecting the prognosis of patients with cirrhosis.

\section{Conclusion}

Our study suggested that overall mortality of patients with alcoholic liver cirrhosis patients may be associated with low SES, which was measured by receiving PA. This association could not be explained by severity of cirrhosis at diagnosis nor dropout rate. Further study is needed to investigate the cause of higher mortality among the low SES population, and potential interventions to the disease burden.

\section{Declarations}

\section{Ethics approval:}

The research was approved by the Institutional Review Board of St Marianna University Hospital (approval number 4995).

\section{Consent to participate:}

Consent to participate was obtained by posting notices of the study in hospital bulleting and opting out.

\section{Consent for publication:}

not applicable

\section{Availability of data and material:}

The datasets used and/or analysed during the current study are available from the corresponding author on reasonable request.

\section{Competing interests:}

The authors declare that they have no competing interests

\section{Funding:}

no funding was provided 


\section{Authors' contributions:}

All authors contributed to study conceptualization and design. Data collection was performed by KM. Data curation was performed by MK. Data analysis was done by $\mathrm{MK}, \mathrm{KI}$ and $\mathrm{MH}$. Supervision was performed by TM. Manuscript was written and edited by $\mathrm{MK}, \mathrm{KI}, \mathrm{MH}, \mathrm{IM}, \mathrm{TT}$ and $\mathrm{CO}$, then reviewed by all authors.

\section{Acknowledgements:}

not applicable

\section{References}

1. Erskine S, Maheswaran R, Pearson T, Gleeson D. Socioeconomic deprivation, urban-rural location and alcohol-related mortality in England and Wales. BMC Public Health. 2010;10.

2. Global Information System on Alcohol and Health [Internet]. [cited 2021 May 19]. Available from: https://www.who.int/data/gho/data/themes/global-information-system-on-alcohol-and-health

3. Testino G, Leone S, Borro P. Alcohol and hepatocellular carcinoma: A review and a point of view. World Journal of Gastroenterology. WJG Press; 2014. p. 15943-54.

4. Sarin SK, Kumar M, Eslam M, George J, Mahtab A, Akbar F, et al. Liver diseases in the Asia-Pacific region: a Lancet Gastroenterology \& Hepatology Commission. The Lancet Gastroenterology \& Hepatology Commission Lancet Gastroenterol Hepatol [Internet]. 2020 [cited 2021 May 28];5:167228. Available from: www.thelancet.com/gastrohep

5. Kposowa AJ, Breault K. Disability Status, Unemployment, and Alcohol-Related Liver Disease (ALD) Mortality: A Large Sample Individual Level Longitudinal Study. Substance Abuse and Rehabilitation. Informa UK Limited; 2021; Volume 12:81-8.

6. Jepsen P, Vilstrup H, Andersen PK, Sørensen HT. Socioeconomic status and survival of cirrhosis patients: A Danish nationwide cohort study. BMC Gastroenterology. 2009;9.

7. Vaz J, Strömberg U, Eriksson B, Buchebner D, Midlöv P. Socioeconomic and marital status among liver cirrhosis patients and associations with mortality: a population-based cohort study in Sweden. BMC Public Health. BioMed Central Ltd; 2020;20.

8. Yuda M. The medical assistance system and inpatient health care provision: Empirical evidence from short-term hospitalizations in Japan. PLoS ONE. Public Library of Science; 2018;13.

9. Khatana SAM, BA, NAS, et al. Association of Medicaid Expansion With Cardiovascular Mortality. . Jama Cardiology. 2019;671-9.

10. Swaminathan S, Sommers BD, Thorsness R, Mehrotra R, Lee Y, Trivedi AN. Association of Medicaid Expansion with 1-Year Mortality among Patients with End-Stage Renal Disease. JAMA - Journal of the American Medical Association. American Medical Association; 2018. p. 2242-50. 
11. Kamath PS, WRH, MM, et al. A model to predict survival in patients with end-stage liver disease. Hepatology. 2001;464-70.

12. Chan AWH, Chan RCK, Wong GLH, Wong VWS, Choi PCL, Chan HLY, et al. New simple prognostic score for primary biliary cirrhosis: Albumin-bilirubin score. Journal of Gastroenterology and Hepatology (Australia). Blackwell Publishing; 2015;30:1391-6.

13. Peng Y, Qi X, Guo X. Child-pugh versus MELD score for the assessment of prognosis in liver cirrhosis a systematic review and meta-analysis of observational studies. Medicine (United States). Lippincott Williams and Wilkins; 2016.

14. Fragaki M, Sifaki-Pistolla D, Orfanoudaki E, Kouroumalis E. Comparative evaluation of albi, meld, and child-pugh scores in prognosis of cirrhosis: Is albi the new alternative? Annals of Gastroenterology. Hellenic Society of Gastroenterology; 2019;32:626-32.

15. Wan SZ, Nie Y, Zhang Y, Liu C, Zhu X. Assessing the Prognostic Performance of the Child-Pugh, Model for End-Stage Liver Disease, and Albumin-Bilirubin Scores in Patients with Decompensated Cirrhosis: A Large Asian Cohort from Gastroenterology Department. Disease Markers. Hindawi Limited; 2020;2020.

16. Pudrovska T, Anikputa B. Early-life socioeconomic status and mortality in later life: An integration of four life-course mechanisms. Journals of Gerontology - Series B Psychological Sciences and Social Sciences. Gerontological Society of America; 2014. p. 451-60.

17. Sugiyama $\mathrm{Y}$, Matsushima M, Yoshimoto H. Association between alcohol consumption/alcohol use disorders and patient complexity: A cross-sectional study. BMJ Open. BMJ Publishing Group; 2020;10.

18. McMaughan DJ, Oloruntoba O, Smith ML. Socioeconomic Status and Access to Healthcare: Interrelated Drivers for Healthy Aging. Frontiers in Public Health. Frontiers Media S.A.; 2020.

19. Mackenbach JP, Kulhánová I, Bopp M, Borrell C, Deboosere P, Kovács K, et al. Inequalities in AlcoholRelated Mortality in 17 European Countries: A Retrospective Analysis of Mortality Registers. PLoS Medicine. Public Library of Science; 2015;12.

\section{Figures}


727 participants were diagnosed as LC in our hospital during year 2006 to 2017

LC patients that did not have alcohol as cause for LC were excluded $(\mathrm{N}=469)$

258 participants that had history of excessive alcohol consumption, and was diagnosed as alcoholic LC by trained hepatologists were left

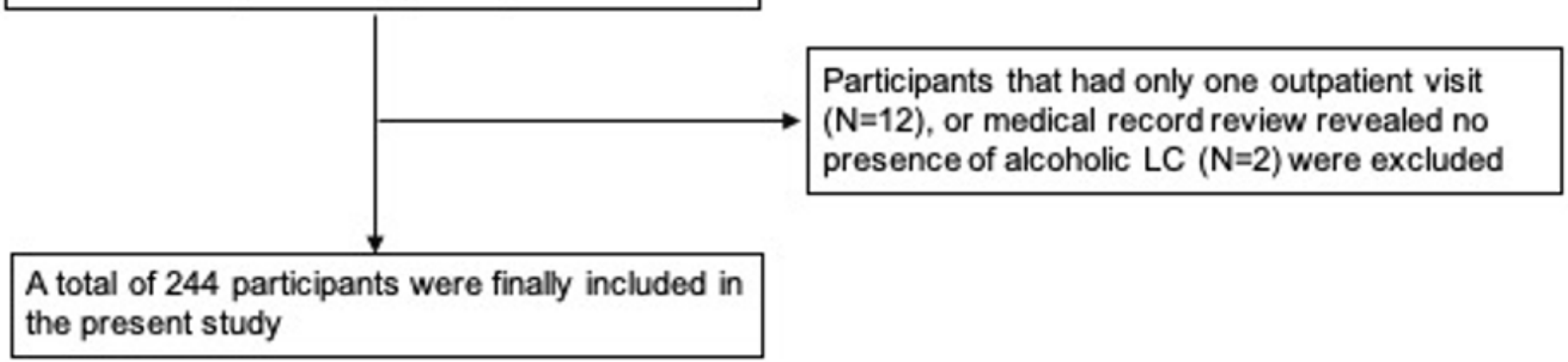

Figure 1

Inclusion and exclusion flowchart. Abbreviations: LC, liver cirrhosis 


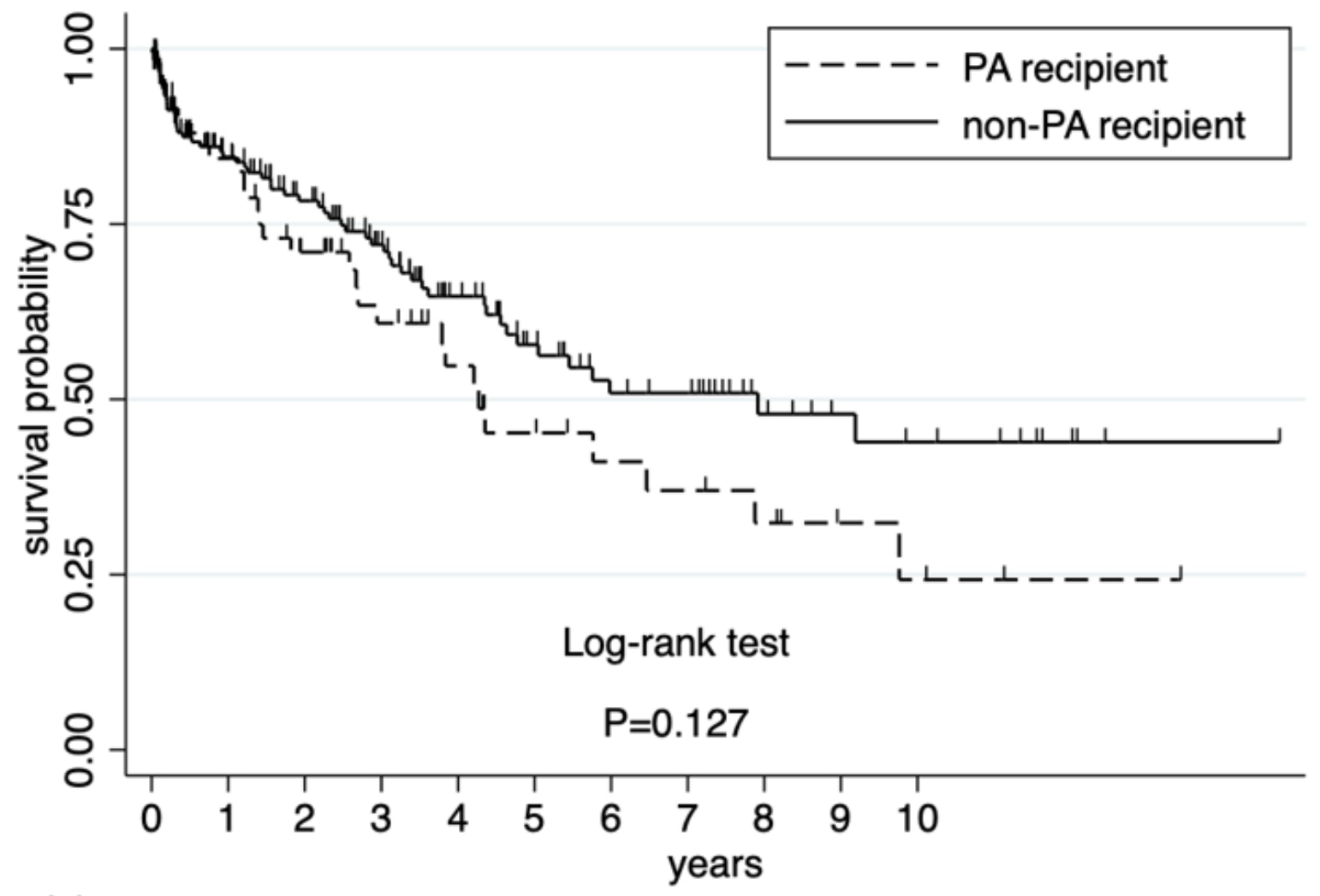

Number at risk

$\begin{array}{llllllllllll}\text { PA recipient } & 61 & 46 & 34 & 24 & 18 & 12 & 10 & 9 & 7 & 4 & 3\end{array}$

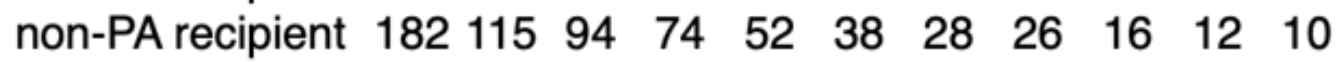

Figure 2

Survival curve for PA recipients and non-PA recipients. Abbreviations: PA, public assistance.

\section{Supplementary Files}

This is a list of supplementary files associated with this preprint. Click to download.

- Supplementarytable1.docx 